\title{
Laser Plasma Accelerators: Towards High Quality Electron Beam
}

\author{
Victor Malka \\ Laboratoire d'Optique Appliquée, ENSTA Paris Tech, CNRS, \\ Ecole Polytechnique Paris Tech, UMR 7639, 91761 Palaiseau \\ France
}

\section{Introduction}

In 1979 Tajima and Dawson, Tajima \& Dawson (1979), on the basis of theoretical work and simulations, have shown that relativistic plasma waves are suitable for the development of compact accelerators. In the proposed schemes, electrons were injected externally and were accelerated through the very high electric field $(\mathrm{GV} / \mathrm{m})$ sustained by relativistic plasma waves driven by lasers, in the laser beatwave regime or in the laser wakefield regime. Several experiments have been performed following their idea and injected electrons at the few $\mathrm{MeV}$ level have indeed been accelerated by electric fields in the GV/m range in a plasma medium using the beat wave and laser wakefield schemes. With the development of more powerful lasers, much higher electric fields were achieved, from few $\mathrm{GV} / \mathrm{m}$ to more than 1 $\mathrm{TV} / \mathrm{m}$, Malka et al. (2002). This fast increase in the laser power has allowed physicists (i) to produce electron beams directly from the interaction of an intense laser with an underdense plasma, i.e. without any external injection and (ii) at the same time to explore new theoretical schemes: forward Raman instability, self-modulated laser wakefield, direct laser acceleration, and forced laser wakefield. In all those experiments, the distribution of the electron was Maxwellian and the beam quality was far from what is required for some applications. These first beams did not compare well to beams produced by conventional accelerators. To control or to shape the electron beam distribution, one has to reduce the injection to a very limited volume of phase space. For external injection, this means that the injected electron beam must have a duration much shorter that the plasma period, i.e. less than ten of femtoseconds. Note that longer electron bunches can be injected and compressed by the plasma wave when they are injected before the laser pulse, Khachatryan et al. (2004). For internal injection, the control of injection can be done either in the bubble/blowout regime using one single laser beam or by colliding two or three laser pulses. In 2004, a major breakthrough came with the experimental demonstration of the bubble/blowout regime when three groups produced for the first time an electron beam with a quasimonoenergetic distribution, Mangles et al. (2004); Geddes et al. (2004); Faure et al. (2004). The bubble regime is reached when the laser power is high enough and when the laser pulse length and waist match the plasma wavelength. When these conditions are met, the laser ponderomotive force expels the plasma electrons radially and leaves a cavitated region behind the pulse. Electrons are progressively injected at the back of this cavity forming a dense electron beam in the cavity. The increasing charge of the forming electron beam progressively reduces the electric value and the injection process eventually stops, leading to the formation of a quasimonoenergetic electron beam. 
A continuous transition between the self-modulated laser wakefield, forced laser wakefield, and bubble regimes has been measured by changing the electron density, i.e., by changing the ratio of the pulse length to the plasma wavelength. The use of two laser beams instead of one offers more exibility and enables one to separate the injection from the acceleration processes. The first laser beam the pump beam (the injection beam) is used to heat electrons during its collision with the pump beam. During the beating, electrons can gain enough momentum to catch the relativistic plasma wave and consequently be trapped and efficiently accelerated. In 2006, stable and tunable quasimonoenergetic electron beams were measured by using two laser beams in the colliding scheme with a counterpropagating geometry, Faure et al. (2006).

\section{Theory and evolution of electron acceleration using laser-plasma interaction}

\subsection{Maxwell equations}

When a laser pulse propagates in a medium, its corresponding electromagnetic wave evolution is described by the Maxwell equations :

$$
\begin{cases}\vec{\nabla} \cdot \vec{E}=\frac{\rho}{\varepsilon_{0}} & \vec{\nabla} \cdot \vec{B}=0 \\ \vec{\nabla} \wedge \vec{E}=-\frac{\partial \vec{B}}{\partial t} & \vec{\nabla} \wedge \vec{B}=\frac{1}{c^{2}} \frac{\partial \vec{E}}{\partial t}+\mu_{0} \vec{j}\end{cases}
$$

where $\vec{E}$ and $\vec{B}$ are the electric and magnetic fields respectively, $\rho$ and $\vec{j}$ describe the medium and are the local density and the current density respectively. $\varepsilon_{0}$ and $\mu_{0}$ are the permittivity and permeability of vacuum. $c$ is the celerity of light. The operator $\vec{\nabla}$ corresponds to the spatial partial derivative vector

$$
\vec{\nabla}=\left(\frac{\partial}{\partial x}, \frac{\partial}{\partial y}, \frac{\partial}{\partial z}\right)
$$

From this set of equations, one obtains the equation of propagation of the electric field. In vacuum $(\rho=0$ and $\vec{j}=\overrightarrow{0})$, this is written :

$$
\nabla^{2} \vec{E}(\vec{x}, t)-\frac{1}{c^{2}} \frac{\partial^{2} \vec{E}}{\partial t^{2}}(\vec{x}, t)=\overrightarrow{0}
$$

In the frequency domain (for the temporal variable), it becomes :

$$
\nabla^{2} \vec{E}(\vec{x}, \omega)+\frac{\omega^{2}}{c^{2}} \vec{E}(\vec{x}, \omega)=\overrightarrow{0}
$$

In a more general case of a propagation in an isotropic medium, one introduces the refractive index $\eta$ in the equation of propagation, which contains the response of the medium

$$
\nabla^{2} \vec{E}(\vec{x}, \omega)+\frac{\omega^{2}}{c^{2}} \eta^{2}(\omega) \vec{E}(\vec{x}, \omega)=\overrightarrow{0}
$$

\subsection{Laser parameters}

In general, one uses potentials to describe these fields. There exist a vector potential $\vec{A}$ and a scalar potential $\Phi$ that verify :

$$
\left\{\begin{array}{l}
\vec{E}=-\vec{\nabla} \Phi-\frac{\partial \vec{A}}{\partial t} \\
\vec{B}=\vec{\nabla} \wedge \vec{A}
\end{array}\right.
$$


These potentials are not described uniquely. It is possible to find other solutions with a gauge transformation. We usually work in Coulomb gauge $\vec{\nabla} \cdot \vec{A}=0$.

In the following, we will use the normalized vector potential $\vec{a}$, defined by :

$$
\vec{a}=\frac{e \vec{A}}{m_{e} c}
$$

where $e$ is the electron charge and $m_{e}$ its mass.

One also introduces the intensity $I$, which is the average of the Poynting vector over an optical cycle :

$$
I=c^{2} \varepsilon_{0}\langle\vec{E} \wedge \vec{B}\rangle_{t}
$$

where brackets design the temporal average on one optical cycle.

\subsection{Gaussian beams}

Short laser pulses delivered by laser systems have a broad spectrum which contains many modes locked in phase. This spectrum is usually described simply by a gaussian envelope, thus also leading to an gaussian temporal envelope, which is close to reality. In the same way, the spatial profile of the laser pulse at the focal plane is also represented by a gaussian function. The electric field has the following form for a linearly polarized pulse :

$$
\vec{E}(r, z, t)=\frac{E}{2} f(r, z) g(t, z) \exp \left[-i\left(k_{0} z-\omega_{0} t\right)\right] \overrightarrow{e_{x}}+\overrightarrow{c c}
$$

Equation (9) contains a carrying envelope with wave number $k_{0}$ and frequency $\omega_{0}$ and spatial and temporal information contained in $f(r, z)$ and $g(t)$ respectively. The following gaussian expressions (10) verify the equation of propagation of the electric field in vacuum in the paraxial approximation. These expressions reproduce accurately the electric field of the laser when the focusing optics have small aperture.

$$
\begin{aligned}
& g(t, z)=\exp \left[-2 \ln 2\left(\frac{t-z / c}{\tau_{0}}\right)^{2}\right] \\
& f(r, z)=\frac{\mathrm{w}_{0}}{\mathrm{w}(z)} \exp \left[-\frac{r^{2}}{\mathrm{w}^{2}(z)}-i \frac{k_{0} r^{2}}{2 R(z)}\right] \exp i \phi(z)
\end{aligned}
$$

where $\tau_{0}$ is the pulse duration at full width at half maximum (FWHM), $\mathrm{w}_{0}$ is the waist of the focal spot (the radius at $1 / e$ of the electric field in the focal plane $z=0) . \phi(z)$ is the Gouy phase. Functions $\mathrm{w}(z)$ and $R(z)$ represent respectively the radius at $1 / e$ of the electric field and the radius of curvature of the wave front. These functions take the following form:

$$
\begin{aligned}
& \mathrm{w}(z)=\mathrm{w}_{0} \sqrt{1+\frac{z^{2}}{Z_{r}^{2}}} \\
& R(z)=z\left(1+\frac{Z_{r}^{2}}{z^{2}}\right)
\end{aligned}
$$

$Z_{r}=\pi \mathrm{w}_{0}^{2} / \lambda_{0}$ is the Rayleigh length. This physical parameter represents the length where the laser intensity on axis has dropped by a factor 2 compared to the intensity in the focal plane $(z=0)$. 
Starting from this expression of the electric field, the following relation exists between the maximal intensity $I_{0}$ and the power $P$ :

$$
I_{0}=\frac{2 P}{\pi \mathrm{w}_{0}^{2}}
$$

with $P=2 \sqrt{\frac{\ln 2}{\pi}} \frac{U}{\tau_{0}} \sim \frac{U}{\tau_{0}}$, where $U$ is the energy contained in the pulse.

Then, the following relation lies the maximal intensity $I_{0}$ and the maximum of the normalized vector potential $a_{0}$

$$
a_{0}=\left(\frac{e^{2}}{2 \pi^{2} \varepsilon_{0} m_{e}^{2} c^{5}} \lambda_{0}^{2} I_{0}\right)^{1 / 2}
$$

When $a_{0}$ exceeds unity, the oscillations of an electron in the laser field become relativistic. In laser plasma accelerators the motion of the electrons is mostly relativistic ${ }^{1}$.

\subsection{Plasma parameters}

A plasma is a state of matter made of free electrons, totally or partially ionized ions and neutral atoms or molecules, the whole medium being globally neutral. Let's assume an initially uniform, non-collisional plasma in which a slab of electron is displaced from the equilibrium position. The restoring force which applies on this electron slab, drives them towards the equilibrium position. For the time scale corresponding to the electron motion, one neglects the motion of the ions because of the inertia. This gives in the end oscillations around the equilibrium position at a frequency called the electron plasma frequency $\omega_{p e}$

$$
\omega_{p e}=\sqrt{\frac{n_{e} e^{2}}{m_{e} \varepsilon_{0}}}
$$

where $n_{e}$ is the unperturbed electron density.

This frequency has to be compared to the laser frequency : if $\omega_{p e}<\omega_{0}$ then the characteristic time scale of the plasma is longer than the optical period of the incoming radiation. The medium can't stop the propagation of the electromagnetic wave. The medium is said to be transparent or under-dense. On the opposite, when $\omega_{p e}>\omega_{0}$ then the characteristic time scale of the electrons is fast enough to adapt to the incoming wave and to reflect totally of partially the radiation. The medium is said to be overdense.

These two domains are separated at frequency $\omega_{0}$, which corresponds to the critical density 2 $n_{c}=\omega_{0}^{2} m_{e} \varepsilon_{0} / e^{2}$

\subsubsection{Electric field of the plasma wave}

One considers now a periodic sinusoidal perturbation of the electron plasma density in a uniform ion layer. Mechanisms responsible for the excitation of the plasma wave will be described in the following section. The density perturbation $\delta n$ is written :

$$
\delta n=\delta n_{e} \sin \left(k_{p} z-\omega_{p} t\right)
$$

where $\omega_{p}$ and $k_{p}$ are the angular frequency and the wave number of the plasma wave.

\footnotetext{
${ }^{1}$ For a visible laser light intensity $I_{0}=3 \times 10^{18} \mathrm{~W} / \mathrm{cm}^{2}$, to which corresponds a $a_{0}=1.3$.

${ }^{2}$ For an wavelength $\lambda_{0}=820 \mathrm{~nm}$, one obtains a critical density of $n_{c}=1.7 \times 10^{21} \mathrm{~cm}^{-3}$
} 
This density perturbation leads to a perturbation of the electric field $\delta \vec{E}$ via the Poisson equation:

$$
\vec{\nabla} \cdot \delta \vec{E}=-\frac{\delta n e}{\varepsilon_{0}}
$$

This gives

$$
\delta \vec{E}(z, t)=\frac{\delta n_{e} e}{k_{p} \varepsilon_{0}} \cos \left(k_{p} z-\omega_{p} t\right) \overrightarrow{e_{z}}
$$

Because we want to describe the electron acceleration to relativistic energies by a plasma wave, we consider now a plasma wave with a phase velocity is close to the speed of light $v_{p}=$ $\omega_{p} / k_{p} \sim c$. Let $E_{0}=m_{e} c \omega_{p e} / e$. The electric field becomes :

$$
\delta \vec{E}(z, t)=E_{0} \frac{\delta n_{e}}{n_{e}} \cos \left(k_{p} z-\omega_{p} t\right) \overrightarrow{e_{z}}
$$

One notice that the electric field is dephased by $-\pi / 4$ with respect to the electron density.

\subsubsection{Lorentz's transform}

Let's now describe what happens to an electron placed in this electric field. The goal is to obtain the required conditions for trapping to occur. The following variables are introduced to describe the electron in the laboratory frame : $z$ the position, $t$ the associated time, $\beta$ the velocity normalized to $c, \gamma=1 / \sqrt{1-\beta^{2}}$ the associated Lorentz's factor. In the frame of the plasma wave, let $z^{\prime}, t^{\prime}, \beta^{\prime}$ and $\gamma^{\prime}$ represent the equivalent quantities.

The frame linked to the plasma wave is in uniform constant translation at speed $v_{p}=\beta_{p} c$. One writes $\gamma_{p}$ the Lorentz's factor associated to this velocity. The Lorentz's transform allows to switch from the laboratory frame to the wave frame :

$$
\left\{\begin{array}{l}
z^{\prime}=\gamma_{p}\left(z-v_{p} t\right) \\
t^{\prime}=\gamma_{p}\left(t-\frac{x}{c}\right) \\
\gamma^{\prime}=\gamma \gamma_{p}\left(1-\vec{\beta} \cdot \overrightarrow{\beta_{p}}\right)
\end{array}\right.
$$

In this new frame, without magnetic field, the electric field remains unchanged $\delta \vec{E}^{\prime}$

$$
\delta \overrightarrow{E^{\prime}}\left(z^{\prime}\right)=\delta \vec{E}(z, t)=E_{0} \frac{\delta n_{e}}{n_{e}} \cos \left(k_{p} z^{\prime} / \gamma_{p}\right) \overrightarrow{e_{z}}
$$

Consequently, in terms of potential, the electric field is derived from potential $\Phi^{\prime}$ defined by

$$
\vec{F}=-e \delta \vec{E}^{\prime} \equiv-\vec{\nabla}^{\prime} \Phi^{\prime}
$$

This leads to

$$
\Phi^{\prime}\left(z^{\prime}\right)=m c^{2} \gamma_{p} \frac{\delta n_{e}}{n_{e}} \sin \left(k_{p} z^{\prime} / \gamma_{p}\right) \equiv m c^{2} \phi^{\prime}\left(z^{\prime}\right)
$$

Finally, one writes the total energy conservation for the particle in this frame compared to the initial energy at the injection time (labelled with subscript 0) :

$$
\gamma^{\prime}\left(z^{\prime}\right)+\phi^{\prime}\left(z^{\prime}\right)=\gamma_{0}^{\prime}\left(z_{0}^{\prime}\right)+\phi_{0}^{\prime}\left(z_{0}^{\prime}\right)
$$

Equation 24 gives the relation between the electron energy and its position in the plasma wave. Figure 1 illustrates the motion of an electron injected in this potential. Finally, we perform the reverse Lorentz's transform to give this energy in the laboratory frame. 
For $\beta^{\prime}>0$, the scalar product in eq. 20 is positive

$$
\gamma=\gamma^{\prime} \gamma_{p}+\sqrt{\gamma^{\prime 2}-1} \sqrt{\gamma_{p}^{2}-1}
$$

For $\beta^{\prime}<0$, scalar product in eq. 20 is negative

$$
\gamma=\gamma^{\prime} \gamma_{p}-\sqrt{\gamma^{\prime 2}-1} \sqrt{\gamma_{p}^{2}-1}
$$

\subsubsection{Electron trajectories}

Figure 1 represents an example of electron trajectory in a plasma wave. In this phase space, the closed orbits correspond to trapped particles. Open orbits represent untrapped electrons, either because the initial velocity is too low, or to high. The curve which separates these two regions is called the separatrix.

This separatrix gives the minimum and maximum energies for trapped particles. This is comparable to the hydrodynamic case, where a surfer has to crawl to gain velocity and to catch the wave. In terms of relativistic factor, $\gamma$ has to belong to the interval $\left[\gamma_{\min } ; \gamma_{\max }\right]$ with :

$$
\left\{\begin{array}{l}
\gamma_{\min }=\gamma_{p}\left(1+2 \gamma_{p} \delta\right)-\sqrt{\gamma_{p}^{2}-1} \sqrt{\left(1+2 \gamma_{p} \delta\right)^{2}-1} \\
\gamma_{\max }=\gamma_{p}\left(1+2 \gamma_{p} \delta\right)+\sqrt{\gamma_{p}^{2}-1} \sqrt{\left(1+2 \gamma_{p} \delta\right)^{2}-1}
\end{array}\right.
$$

where $\delta=\delta n_{e} / n_{e}$ is the relative amplitude of the density perturbation.

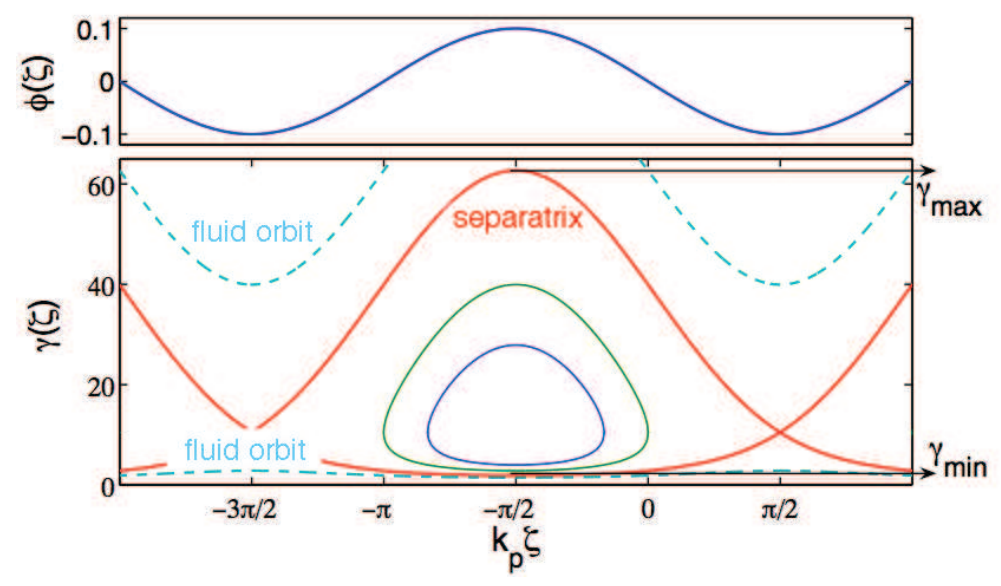

Fig. 1. Up: Potential in phase space. Down: Trajectory of an electron injected in the potential of the plasma wave in the frame of the wave with the fluid orbit (dashed line), the trapped orbit and in between in red the separatrix.

One deduces that the maximum energy gain $\Delta W_{\max }$ for a trapped particle is reached for a closed orbit with maximum amplitude. This corresponds to the injection at $\gamma_{\min }$ on the separatrix and its extraction at $\gamma_{\max }$. The maximum energy gain is then written

$$
\Delta W_{\max }=\left(\gamma_{\max }-\gamma_{\min }\right) m c^{2}
$$


For an electron density much lower than the critical density $n_{e} \ll n_{c}$, one has $\gamma_{p}=\omega_{0} / \omega_{p} \gg 1$ and

$$
\Delta W_{\max }=4 \gamma_{p}^{2} \frac{\delta n_{e}}{n_{e}} m c^{2}
$$

For electron travelling along the separatrix, the time necessary to reach maximal energy is infinite because there exist a stationnary point at energy $\gamma_{p}$. On other closed orbits, the electron successively gains and looses energy during its rotation of the phase space. In order to design an experiment, one needs an estimation of the distance an electron travels before reaching maximal energy gain. This length, which is called the dephasing length $L_{\text {deph }}$, corresponds to a phase rotation of $\lambda_{p} / 2$ in the phase space. In order to have a simple analytical estimation, one needs to assume that the energy gain is small compared to the initial energy of the particle and that the plasma wave is relativistic $\gamma_{p} \gg 1$, then the dephasing length is written

$$
L_{\text {deph }} \sim \gamma_{p}^{2} \lambda_{p}
$$

This concept of dephasing length in a 1D case can be refined in a bi-dimensional case. Indeed, if one also takes into account the transverse effects of the plasma wave, this one is focusing or defocusing for the electrons along their acceleration, Mora (1992). Because these transverse effects are shifted by $\lambda_{p} / 4$ with respect to the pair acceleration/deceleration, the distance over which the plasma wave is both focusing and accelerating is restricted to a rotation of $\lambda_{p} / 4$ in phase space, which decreases by a factor 2 the dephasing length from eq. 30.

$$
L_{\text {deph }}^{2 \mathrm{D}} \sim \gamma_{p}^{2} \lambda_{p} / 2
$$

In these formulas, one has considered a unique test electron, which has no influence on the plasma wave. In reality, the massive trapping of particles modifies electric fields and distorts the plasma wave. Finally, this linear theory is difficult to apply to highly non-linear regimes which are explored experimentally. Some non-linear effects concerning short pulses are described in the next section. Nonetheless, these formulas are usefull to scale the experiments.

\subsection{Non-linear effects}

\subsubsection{Ponderomotive force}

Let's take a non-relativistic electron for a short while. In a laser field with a weak intensity, the average position of an electron is constant. If one only keeps linear terms in fluid equation there remains, Kruer (1988):

$$
{\frac{\partial \vec{v}_{e}}{\partial t}}^{(l)}=-\frac{e}{m_{e}} \vec{E}
$$

The electron directly varies with the electric field. Let's consider now a laser pulse slightly more intense, so that the electron velocity becomes slightly non linear $\vec{v}_{e}={\overrightarrow{v_{e}}}^{(l)}+{\overrightarrow{v_{e}}}^{(n l)}$ with $\left\|\vec{v}_{e}^{(n l)}\right\| \ll\left\|\vec{v}_{e}^{(l)}\right\|$. The second order terms satisfy the following equation

$$
\frac{\partial{\overrightarrow{v_{e}}}^{(n l)}}{\partial t}=-\left({\overrightarrow{v_{e}}}^{(l)} \cdot \vec{\nabla}\right){\overrightarrow{v_{e}}}^{(l)}-\frac{e}{m_{e}}\left({\overrightarrow{v_{e}}}^{(l)} \wedge \vec{B}\right)
$$

By keeping the low frequency component of the equation of motion, i.e. by averaging over an optical cycle, one obtains

$$
m_{e} \frac{\partial\left\langle\vec{v}_{e}^{(n l)}\right\rangle_{t}}{\partial t}=-\frac{\vec{\nabla} I}{2 c n_{c}} \equiv \vec{F}_{p}
$$


$\vec{F}_{p}$ is called the ponderomotive force. This force repels charged particles from regions where the laser intensity gradient is large. This ponderomotive force ${ }^{3}$ derives from a ponderomotive potential which is written as follow

$$
\phi_{p}=\frac{I}{2 c n_{c}}=\frac{e^{2} E^{2}}{4 m_{e} \omega_{0}^{2}}
$$

\subsubsection{Laser self-focusing}

For a laser intensity above $10^{19} \mathrm{~W} / \mathrm{cm}^{2}$, the motion of an electron in an intense laser field becomes relativistic. In this case, local properties of the medium vary as function of the laser intensity. In particular, the refractive index in the equation of propagation (eq. 5) depends on laser intensity $\eta(I)=\eta_{0}+\eta_{2} I$. The plasma medium acts as a focusing lens for the electromagnetic field of the laser. If one considers only the relativistic contribution, the critical power for self-focusing $P_{c}$ for a linearly polarized laser pulse ${ }^{4}$ is written, Sprangle et al. (1987)

$$
P_{c}=\frac{8 \pi \varepsilon_{0} m_{e}^{2} c^{5}}{e^{2}} \frac{n_{c}}{n_{e}}
$$

This formula doesn't account for other phenomena which also modify the refractive index : the plasma wave, the ponderomotive effect on the electrons, the ion channel created by a long prepulse. For instance, the plasma wave tends to defocus the laser pulse, which might prevent the pulse from self-focusing at $P_{c}$, Ting et al. (1990). Then, because of an electron density bump at the front of the plasma wave, the laser field in the first plasma bucket can't self-focus , Sprangle \& Esarey (1992). Consequently, the laser pulse tends to erodes by the front. In particular, this theory predicts that it's not possible for a laser pulse shorter than the plasma wavelength to remain self-focused.

In reality, current experiments use very intense laser pulses $a_{0} \gg 1$ and density perturbations are not linear anymore. Then, consequences on the self-focusing of very short laser pulses are less obvious.

\section{Acceleration mechanisms}

At first glance, the electromagnetic field associated to the laser doesn't seem a good solution to accelerate electrons: the electric field is mainly transverse to the propagation of the wave and its direction alternates every half period of the oscillation. Acceleration mechanisms presented here require an intermediary : the plasma wave. This one is excited by the laser pulse and allows to create a longitudinal electrostatic field favourable to the acceleration of electrons. The general diagram is represented on Fig. 2.

In section 2.4.1, a simple model of the electron acceleration in a plasma wave has been presented. Now, the link between the electromagnetic field of the laser and the plasma wave has to be described. Several mechanisms have been developed to excite a large-amplitude plasma wave. These acceleration mechanisms have evolved as the laser pulse duration shortened and maximal intensity increased. Initially, the acceleration was well described by linear formulas. Then, as the intensity increased, non-linear mechanisms have appeared (Raman instability ,Drake et al. (1974), relativistic self-focusing, Mori et al. (1988), relativistic

\footnotetext{
${ }^{3}$ For an intensity $I_{0}=1 \times 10^{19} \mathrm{~W} / \mathrm{cm}^{2}$ and a wavelength $1 \mu \mathrm{m}$, one obtains a ponderomotive potential of $\phi_{p}=1 \mathrm{MeV}$

${ }^{4}$ For an electron density $n_{e}=10^{19} \mathrm{~cm}^{-3}$, for a laser wavelength $\lambda_{0}=1 \mu \mathrm{m}$, one obtains a critical power $P_{c}=2 \mathrm{TW}$
} 


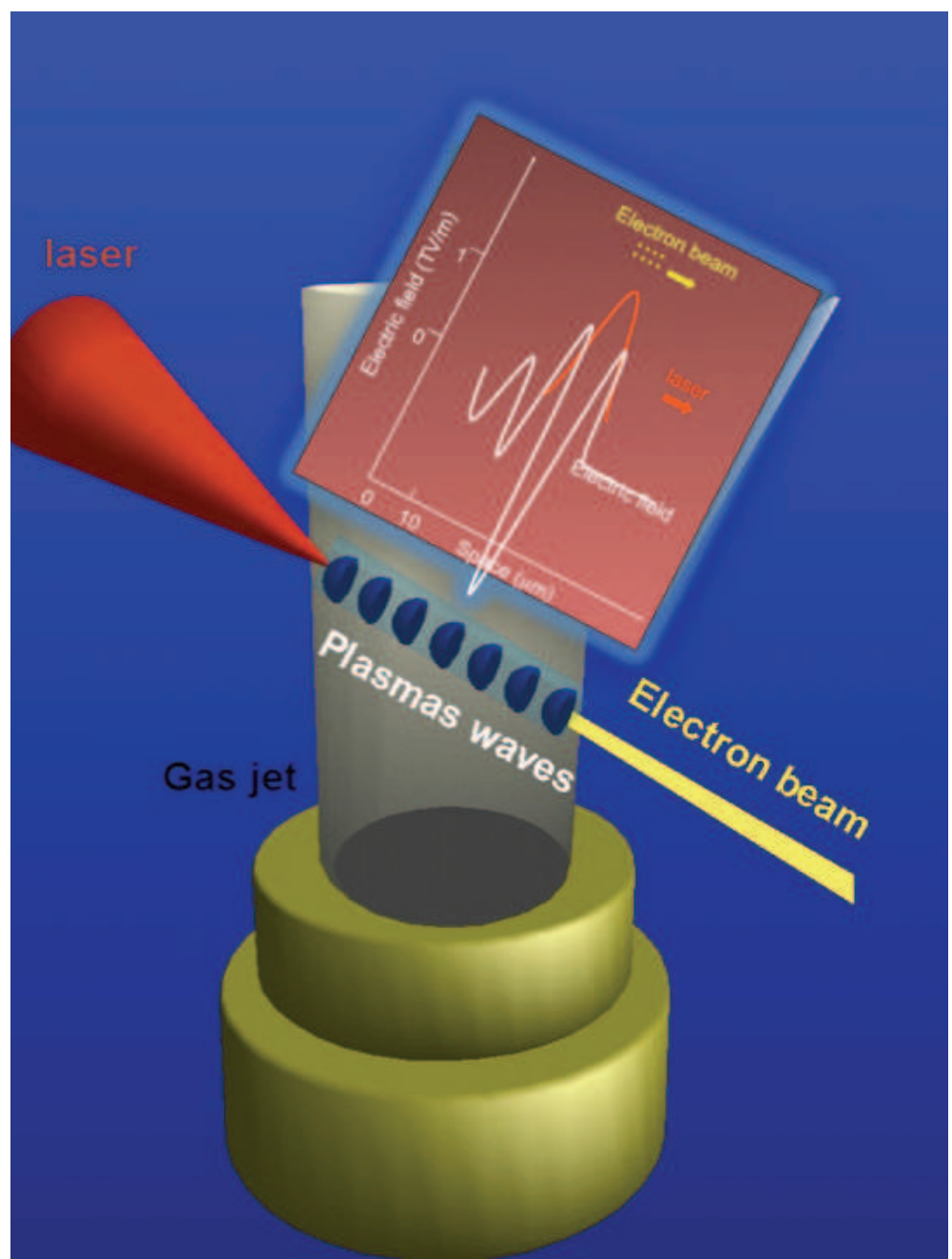

Fig. 2. Principle of laser-plasma acceleration : from the interaction of an intense laser pulse with a gas jet, one obtains an electron beam at the output. 
self-modulation ,McKinstrie \& Bingham (1992)) which allowed to reach even higher electric fields and particle beams with unique properties.

\subsection{Linear regime}

\subsubsection{Laser wakefield}

Acceleration in a laser wakefield has been introduced by Tajima and Dawson, Tajima \& Dawson (1979). The perturbed electron density driven by the laser pulse is favourable to the acceleration of particles. The electron density profile obtained behind a gaussian laser pulse has been reported for $a_{0} \ll 1$, Gorbunov \& Kirsanov (1987). For a linearly polarized laser pulse with full width at half maximum (FWHM) $\sqrt{2 \ln 2} L$ (in intensity), the normalized vector potential is written ${ }^{5}$ :

$$
a^{2}(z, t)=a_{0}^{2} \exp \left[-\left(\frac{k_{0} z-\omega_{0} t}{k_{p} L}\right)^{2}\right]
$$

In this case, the associated electric field is

$$
\vec{E}(z, t)=E_{0} \frac{\sqrt{\pi} a_{0}^{2}}{4} k_{p} L \exp \left(-k_{p}^{2} L^{2} / 4\right) \cos \left(k_{0} z-\omega_{0} t\right) \overrightarrow{e_{z}}
$$

Equation 38 explicitly shows the dependence of the amplitude of the wave with the length of the exciting pulse. In particular, the maximal value for the amplitude is obtained for a length $L=\sqrt{2} / k_{p}$ (see Fig. 3).

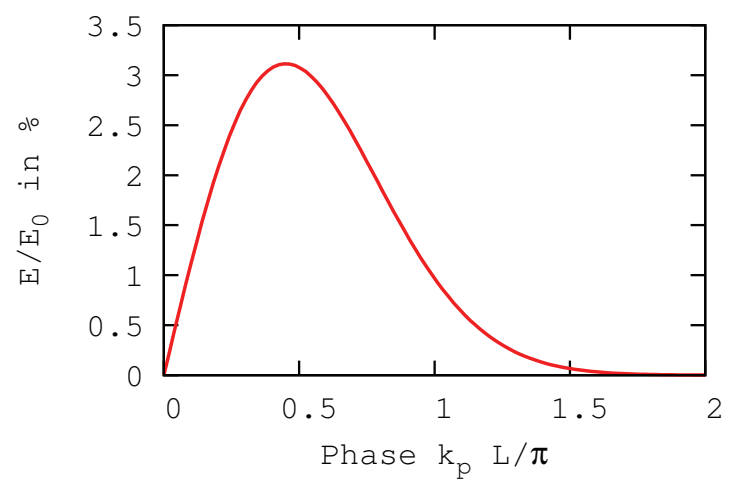

Fig. 3. Amplitude of the electric field as function of the length of a gaussian laser pulse for a normalized vector potential $a_{0}=0.3$.

In figure 4 the density perturbation and the corresponding electric field produced by a $30 \mathrm{fs}$ laser pulse at low intensity $I_{\text {laser }}=3 \times 10^{17} \mathrm{~W} / \mathrm{cm}^{2}$ are shown. One can note that in the linear regime the electric field has sinusoidal shape and reach maximal values of a few $\mathrm{GV} / \mathrm{m}$.

\footnotetext{
${ }^{5}$ For an electron density $n_{e}=10^{19} \mathrm{~cm}^{-3}$, the optimal pulse duration equals $L=2.4 \mu \mathrm{m}$ (equivalent to a pulse duration $\tau=8 \mathrm{fs}$ ). For $a_{0}=0.3$, the maximal electric field is $E=10 \mathrm{GV} / \mathrm{m}$
} 


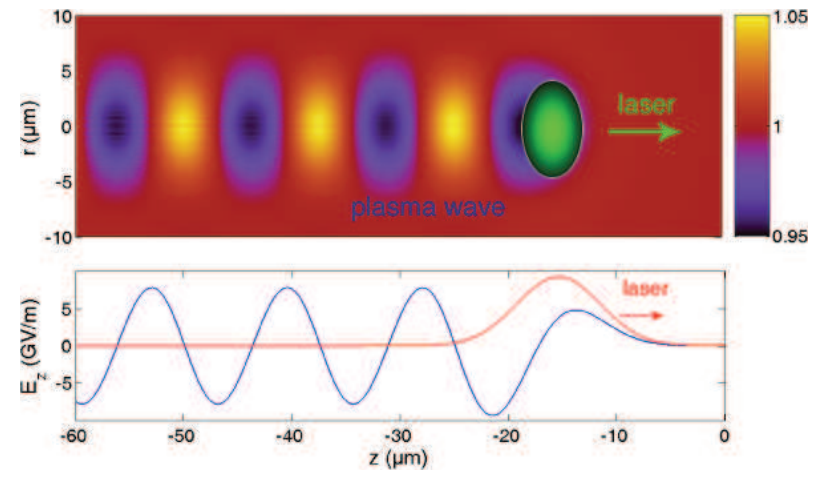

Fig. 4. density perturbation (top) electric field (bottom) produced in the linear regime.

\subsubsection{Non linear regime}

Thanks to the development of laser systems with a high power and a short pulse duration non linear plasma waves can be produced. In the non linear regime the laser pulse excites at resonance plasma wave with much higher amplitude to which corresponds electric field 100 times larger than in the linear regime. One can notice on figure 5 that the radial density perturbation has a horse shoe behavior with bent wakes. As $a_{0}$ grows, wakes become steeper and the wave front becomes curved due to the relativistic shift of the plasma frequency.

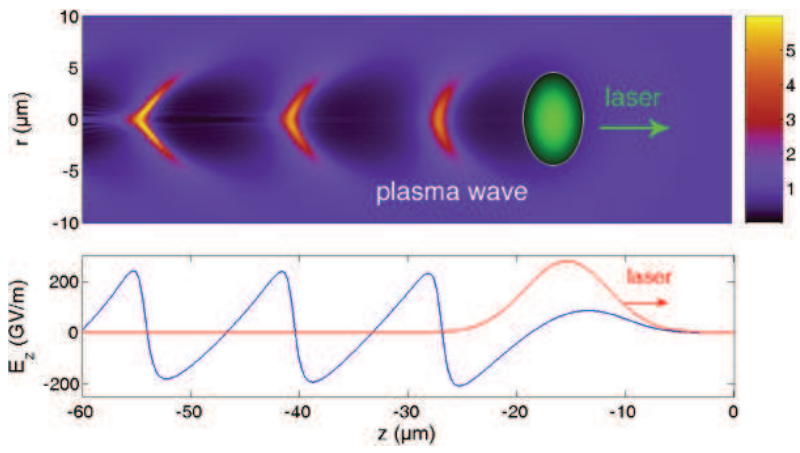

Fig. 5. density perturbation (top) electric field (bottom) produced in the non linear regime.

\subsection{Self-injection}

\subsubsection{Self modulated wakefield}

When the laser power exceeds the critical power for relativistic self-focusing, it temporal shape can be modulated during the propagation in the plasma medium. For laser pulse longer than the plasma wavelength the pulse can be "sausaged" into shorter pulses which excite in a resonant way the relativistic plasma waves. These effects that have been predicted of the basis of numerical simulations, Andreev et al. (1992); Antonsen \& Mora (1992); Sprangle \& Esarey (1992) are illustrated on Fig. 6. This mechanism, which is very similar to Forward Raman Scattering instability, can be described as the decomposition of an electromagnetic wave into a plasma wave an a frequency shifted electromagnetic wave. 

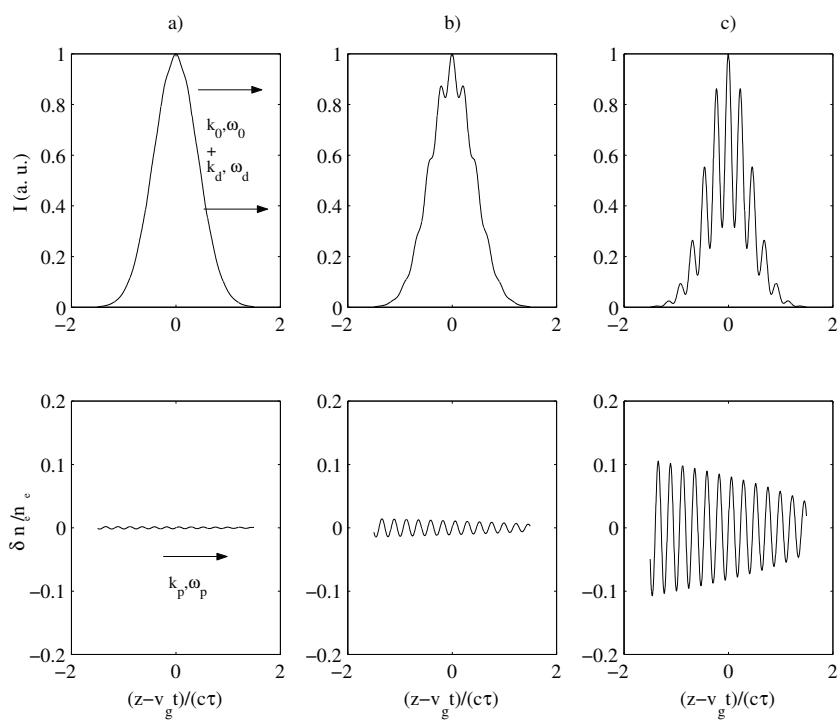

Fig. 6. Self-modulation of the laser envelope and coupling with the plasma wave amplitude. Initially, the laser propagates on a plasma density perturbation (a). This modulates the laser envelope, which increases the coupling with the plasma wave, the amplitude of which increases (b). Finally, the self-modulation mechanism generates a train of laser pulses spaced by a plasma wavelength, which resonantly excites a large amplitude plasma wave (c).

During experiments carried out in England in 1994, Modena et al. (1995), the amplitude of the plasma waves reached the wavebreaking limit, where electrons initially belonging to the plasma wave are self-trapped and accelerated to high energies. The fact that the external injection of electrons in the wave is no longer necessary is a major improvement. Electron spectrum extending up to $44 \mathrm{MeV}$ have been measured during this experiment. This regime has also been reached for instance in the United States at CUOS, Umstadter et al. (1996), at NRL, Moore et al. (2004). However, because of the heating of the plasma by these relatively "long" pulses, the wave breaking occurred well before reaching the cold wave breaking limit, which limited the maximum electric field to a few $100 \mathrm{GV} / \mathrm{m}$. The maximum amplitude of the plasma wave has also been measured by Thomson scattering to be in the range $20-60 \%$, Clayton et al. (1998).

\subsubsection{Forced wakefield}

These unique properties of laser-plasma interaction at very high intensity, previously explored only on very large infrastructures, became accessible for smaller systems, fitted to university laboratories. These laser systems, also based on chirped pulse amplification, Strickland \& Mourou (1985) and using here Titanium Sapphire crystals, fit in a room of several tens of meters square and deliver on-target energy of 2-3 J in $30 \mathrm{fs}$. This corresponds to $100 \mathrm{TW}$-class laser systems which can deliver an intensity of a few $10^{19} \mathrm{~W} / \mathrm{cm}^{2}$ after focusing. Many publications have shown that these facilities which deliver a modest energy and operate at a high repetition rate, can produce energetic electron beams with a quality higher than 


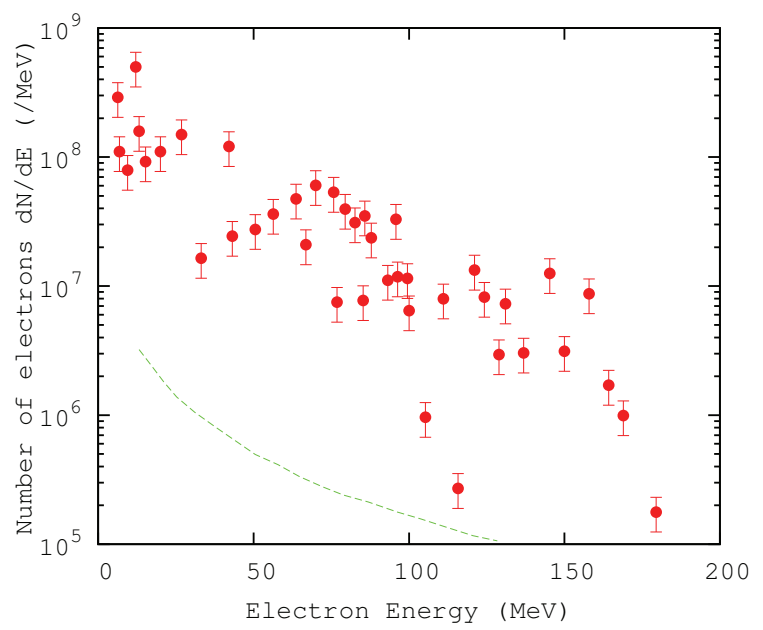

Fig. 7. Typical electron spectrum obtained at $n_{e}=7.5 \times 10^{18} \mathrm{~cm}^{-3}$ with a 1 J-30fs laser pulse focused down to a waist of $\mathrm{w}_{0}=18 \mu \mathrm{m}$. The dashed line corresponds to the detection threshold

larger facilities. For instance, using the laser from "Salle Jaune" at LOA, electrons have been accelerated to $200 \mathrm{MeV}$ in $3 \mathrm{~mm}$ of plasma, Malka et al. (2002). The mechanism involved is called forced laser wakefield to distinguish it from the self-modulated regime.

Indeed, thanks to short laser pulses, the heating of the plasma in the forced laser wakefield is significantly lower than in the self-modulated wakefield. This allows to reach much higher plasma wave amplitudes and also higher electron energies. Thanks to a limited interaction between the laser and the accelerated electrons, the quality of the electron beam is also improved. The measurement of the normalized transverse emittance has given values comparable to those obtained with conventional accelerators with an equivalent energy (normalized rms emittance $\varepsilon_{n}=3 \pi \mathrm{mm}$.mrad for electrons at $55 \pm 2 \mathrm{MeV}$ ), Fritzler et al. (2004). Electron beams with maxwellian spectral distributions (exponential decay, see Fig. 7), generated by ultra-short laser pulses, have been produced in many laboratories in the world : at MPQ in Germany, Gahn et al. (1999), at LOA in France, Malka et al. (2001), at NERL in Japan, Hosokai et al. (2003), and at LBNL in USA, Leemans et al. (2004) for instance.

\subsubsection{Bubble regime}

More recently, theoretical work based on 3D PIC simulations have shown the existence of a robust acceleration mechanism called the bubble regime, Pukhov \& Meyer-ter-Vehn (2002). In this regime, the dimensions of the focused laser are shorter than the plasma wavelength in longitudinal and also transverse directions. Thus, the laser pulse looks like a ball of light with a radius smaller than $10 \mu \mathrm{m}$. If the laser energy contained in this volume is high enough, the ponderomotive force of the laser expels efficiently electrons from the plasma radially, which forms a cavity free from electrons behind the laser, surrounded by a dense region of electrons. Behind the bubble, electronic trajectories intersect each other. A few electrons are injected in the cavity and accelerated along the laser axis, thus creating an electron beam with radial and longitudinal dimensions smaller than those of the laser (see Fig. 8). 


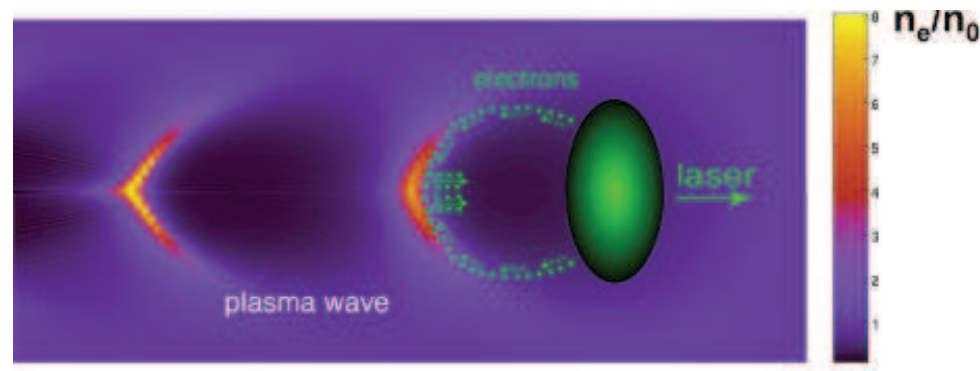

Fig. 8. Acceleration principle in the bubble regime. Electrons circulated around the cavitated region before to be trapped and accelerated at the back of the laser pulse

The signature of this regime is a quasi monoenergetic electron distribution. This contrasts with previous results reported on electron acceleration using laser-plasma interaction. This properties comes from the combination of several factors :

- The electron injection is different from that in the self-modulated or forced regimes. Injection doesn't occur because of the breaking of the accelerating structure. It is localized at the back of the cavity, which gives similar initial properties in the phase space to injected electrons.

- The acceleration takes place in a stable structure during propagation, as long as the laser intensity is strong enough.

- Electrons are trapped behind the laser, which suppresses interaction with the electric field of the laser.

- Trapping stops automatically when the charge contained in the cavity compensates the ionic charge.

- The rotation in the phase-space also leads to a shortening of the spectral width of the electron beam, Tsung et al. (2004).

Several laboratories have obtained quasi monoenergetic spectra : in France, Faure et al. (2004) with a laser pulse shorter than the plasma period, but also with pulses longer than the plasma period in England, Mangles et al. (2004), in the United States, Geddes et al. (2004), then in Japan, Miura et al. (2005) and in Germany, Hidding et al. (2006). The interest of such a beam is important for applications : it is now possible to transport and to refocus this beam by magnetic fields. With a maxwellian-like spectrum, it would have been necessary to select an energy range for the transport, which would have decreased significantly the electron flux. Electrons in the $\mathrm{GeV}$ level were also observed in this regime using in a uniform plasma, Hafz et al. (2008) or in plasma discharge, i.e, a plasma with a parabolic density profile, Leemans et al. (2006) with a more powerful laser which propagates at high intensity over a longer distance.

\subsubsection{Colliding laser pulses scheme}

The control of the parameters of the electron beam (such as the charge, energy, and relative energy spread) is a crucial issue for many applications. In the colliding scheme successfully demonstrated at LOA, it has been shown that not only these issues were addressed but also that a high improvement of the stability was achieved. In this scheme, one laser beam is used to create the relativistic plasma wave, and a second laser pulse which when it collides with 

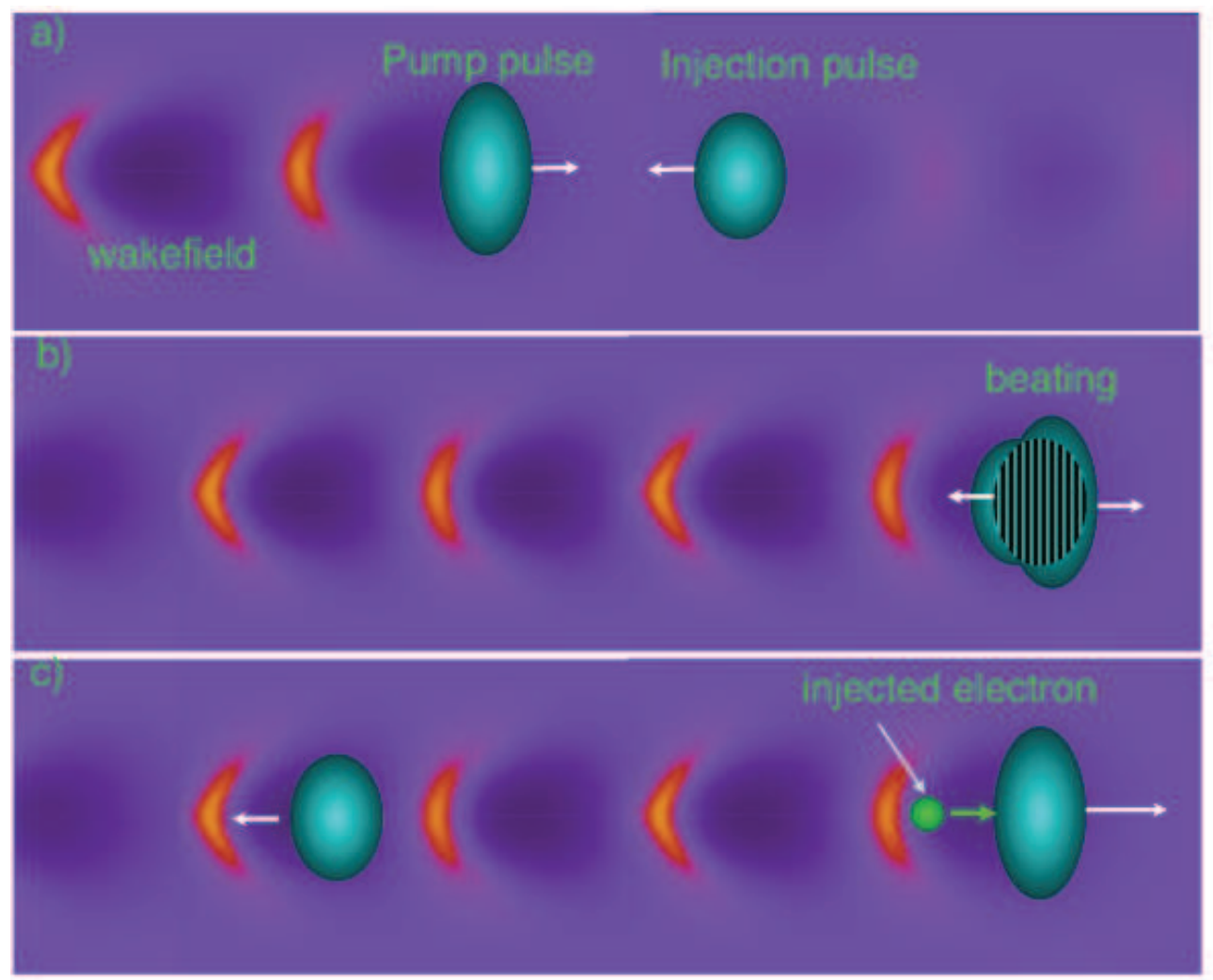

Fig. 9. Principle of injection in the counterpropagating colliding pulse scheme. (1) The two laser pulses have not collided yet; the pump pulse drives a plasma wake (2) The pulses collide and their interference sets up a beatwave that preaccelerates electrons. (3) preaccelerated electrons are trapped and further accelerated in the wake.

the main pulse, creates a standing wave which heats locally electrons of the plasma. The scheme of principle of the colliding laser pulses is shown in Fig 9. The control of the heating level gives not only the number of electrons which will be trapped and accelerated but also the volume of phase space, or in other words, the energy spread of the injected electrons bunch. In the pioneer work of E. Esarey et al., Esarey et al. (1997), a fluid model was used to describe the evolution of the plasma wave whereas electrons were described as test particles. Electron trajectories in the beatwave as well as their energy gain were derived analytically from theory in the case of laser pulses with circular polarization. It has been shown that this approach fails to describe quantitatively the physics occurring at the pulse collision, Rechatin et al. (2007). In the fluid approach, the electron beam charge has been found to be one order of magnitude greater than the one obtained in PIC simulations. For a correct description of injection, one has to describe properly (i) the heating process, e.g. kinetic effects and their consequences on the dynamics of the plasma wave during the beating of the two laser pulses, (ii) the laser pulse evolution which governs the dynamics of the relativistic plasma waves, 
Davoine et al. (2008). New unexpected feature have shown that heating mechanism can be achieved when the two laser pulses are crossed polarized. The stochastic heating can be explained by the fact that for high laser intensities, the electron motion becomes relativistic which introduces a longitudinal component through the $\mathbf{v} \times \mathbf{B}$ force. This relativistic coupling makes it possible to heat electrons. Thus, the two perpendicular laser fields couple through the relativistic longitudinal motion of electrons. The heating level is modified by tuning the intensity of the injection laser beam or by changing the relative polarization of the two laser pulses. This consequently changes the volume in the phase space and therefore the charge and the energy spread of the electron beam. When the pulses have the same polarization, electrons are trapped spatially in the beatwave and can not sustain the collective plasma oscillation inducing a strong inhibition of the plasma wave which persists after the collision. When the polarizations are crossed, the motion of electrons is only slightly disturbed compared to their fluid motion, and the plasma wave is almost unaffected during the collision, which tends to facilitate trapping. In addition to enhance stability, tuning the electron beam energy can be achieved by adjusting the position of the collision in the gas jet, Faure et al. (2006). The collision point can be modied by simply changing the delay between the two laser pulses. If the lasers collide at the entrance of thegas jet, electrons will be injected at an early stage and they can be accelerated over the whole gas jet length $(2 \mathrm{~mm})$. Thus, their energy will be high. On the contrary, injection at the exit of the gas jet will limit the acceleration length and will lead to a low energy beam. The robustness of this scheme has also allow to carry out very accurate studies of the dynamic of electric field in presence of high current electron beam. This beam loading effect has been used to reduce the relative energy spread of the electron beam. We have shown that there is an optimal load can flattened the electric field in such suitable way that a very small, $1 \%$, relative energy spread electron beam can outcome form the target as shown on figure 10 . In this case, the more energetic electrons are slightly slow down and accelerated at the same energy that the slower one. In case of lower charge, this effect doesn't play any role and the energy spread depend mainly of the heating volume. For higher charge, the load is too high and the most energetic electrons slow down too much that they get energies even smaller that the slower one, Rechatin et al. (2009), increasing the relative energy spread. The optimal load was observed experimentally in agreement with full 3D PIC simulations, its corresponds to a current in the 20-40 kA. The decelerating electric field due to the electron beam was found to be in the $\mathrm{GV} / \mathrm{m} / \mathrm{pC}$.

\section{Future of the laser-based acceleration}

Conventional accelerator technology has progressed through a long road paved by scientific challenges. A recent example is the development of superconductivity for high current acceleration in RF cavity, which has required tens of years of theoretical investigations and experimentations to understand the physical processes and finally to control the technology which has been successfully used in accelerators such as LEP/LHC (CERN), or HERA (DESY-Hamburg). Laser plasma accelerator researches follow the same road paved with many successful (and unsuccessful) experiments. Thanks to this pioneering works and judging from the incredible results achieved over the last three years, the time has come where a technological approach has to be considered. Two stages laser plasma accelerators schemes should allow the development of few $\mathrm{GeV}$ electron beam with a small relative energy spread and emittance, Malka et al. (2006). In parallel, fundamental and experimental research should of course be pursued to explore new regimes and to validate theories and numerical codes. The improvement of the laser plasma interaction with the evolution of short-pulse laser 


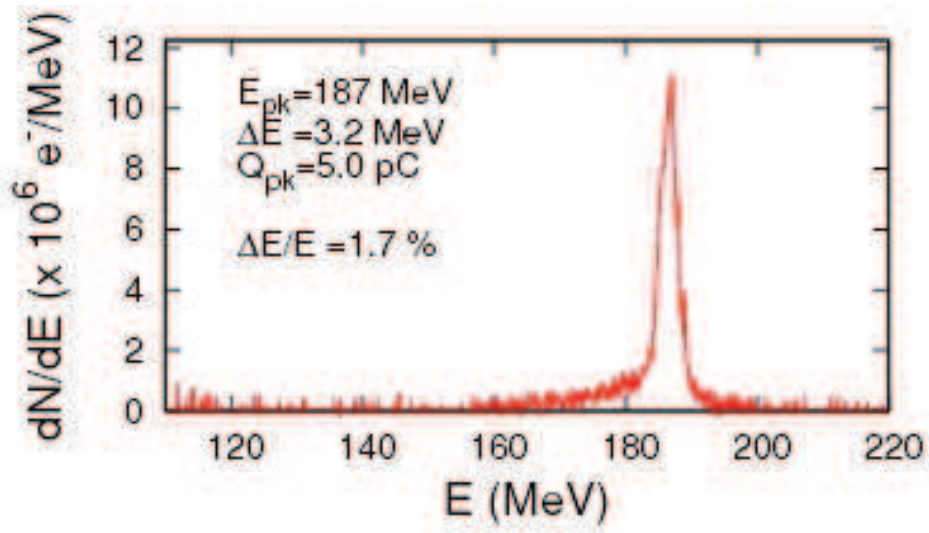

Fig. 10. Typical spectrum obtained in the colliding laser pulse scheme showing a $1 \%$ relative energy spread.

technology, a field in rapid progress, will still improve this new and very promising approach which potential societal applications in material science, medicine, chemistry and radiobiolgy, Malka et al. (2008). The ultra short duration (few fs) of the electron beam, Rechatin et al. (2010), and consequently his very high current (few $\mathrm{kA}$ ) comparable to the one delivers at SLAC for LCLS experiment, where very bright $X$ rays beam was produced by saturating the gain of their free electron laser, indicate that laser plasma accelerators should play a significant role in the compactness of free electron laser design and achievement.

\section{Acknowledgements}

I acknowledge warmly J. Faure, Y. Glinec, C. Rechatin, X. Davoine, E. Lefebvre, and A. Lifschitz who have largely contributed during this last decade to the work I presented in this book chapter. I also acknowledge the support of the European Research Council for funding the PARIS ERC project (contract number 226424), of the European Community-Research Infrastructure Activity under the FP6 and of European Community "Structuring the European Research Area" program (CARE, contract number RII3-CT-2003-506395), of the European Community-New and Emerging Science and Technology Activity under the FP6 "Structuring the European Research Area" program (project EuroLEAP, contract number 028514), and of the French national agency ANR-05-NT05-2-41699 "ACCEL1".

\section{References}

Andreev, N. E., Gorbunov, L. M., Kirsanov, V. I., Pogosova, A. A. \& Ramazashvili, R. R. (1992). Resonant excitation of wakefields by a laser pulse in a plasma, JETP Lett 55: 571.

Antonsen, Jr., T. M. \& Mora, P. (1992). Self-focusing and Raman scattering of laser pulses in tenuous plasmas, Phys. Rev. Lett. 69(15): 2204-2207.

Clayton, C. E., Tzeng, K.-C., Gordon, D., Muggli, P., Mori, W. B., Joshi, C., Malka, V., Najmudin, Z., Modena, A., Neely, D. \& Dangor, A. E. (1998). Plasma wave generation in a self-focused channel of a relativistically intense laser pulse, Phys. Rev. Lett. 81(1): 100. 
Davoine, X., Lefebvre, E., Faure, J., Rechatin, C., Lifschitz, A. \& Malka, V. (2008). Simulation of quasimonoenergetic electron beams produced by colliding pulse wakefield acceleration, Phys. Plasmas 15(11): 113102.

Drake, J. F., Kaw, P. K., Lee, Y. C., Schmidt, G., Liu, C. S. \& Rosenbluth, M. N. (1974). Parametric instabilities of electromagnetic waves in plasmas, Phys. Fluids 17(4): 778.

Esarey, E., Sprangle, P., Krall, J. \& Ting, A. (1997). Self-focusing and guiding of short laser pulses in ionizing gazes and plasmas, IEEE J. Quant. Electron. 33(11): 1879-1914.

Faure, J., Glinec, Y., Pukhov, A., Kiselev, S., Gordienko, S., Lefebvre, E., Rousseau, J.-P., Burgy, F. \& Malka, V. (2004). A laser-plasma accelerator producing monoenergetic electron beams, Nature 431: 541-544.

Faure, J., Rechatin, C., Norlin, A., Lifschitz, A., Glinec, Y. \& Malka, V. (2006). Controlled injection and acceleration of electrons in plasma wakefields by colliding laser pulses., Nature 444: 737-739.

Fritzler, S., Lefebvre, E., Malka, V., Burgy, F., Dangor, A. E., Krushelnick, K., Mangles, S. P. D., Najmudin, Z., Rousseau, J.-P. \& Walton, B. (2004). Emittance measurements of a laser-wakefield-accelerated electron beam, Phys. Rev. Lett. 92(16): 165006.

Gahn, C., Tsakiris, G. D., Pukhov, A., Meyer-ter-Vehn, J., Pretzler, G., Thirolf, P., Habs, D. \& Witte, K. J. (1999). Multi-MeV electron beam generation by direct laser acceleration in high-density plasma channels, Phys. Rev. Lett. 83(23): 4772-4775.

Geddes, C. G. R., Tóth, C., van Tilborg, J., Esarey, E., Schroeder, C. B., Bruhwiler, D., Nieter, C., Cary, J. \& Leemans, W. P. (2004). High quality electron beams from a laser wakefield accelerator using plasma-channel guiding, Nature 431: 538-541.

Gorbunov, L. M. \& Kirsanov, V. I. (1987). Excitation of plasma waves by an electromagnetic wave packet, Sov. Phys. JETP 66: 290-294.

Hafz, N., Jeong, T. M., Choi, I. W., Lee, S. K., Pae, K. H., Kulagin, V. V., Sung, J. H., Yu, T. J., Hong, K.-H., Hosokai, T., Cary, J. R., Ko, D.-K. \& Lee, J. (2008). Stable generation of $\mathrm{GeV}$-class electron beams from self-guided laserplasma channels, Nature Photonics 2: 571-577.

Hidding, B., Amthor, K.-U., Liesfeld, B., Schwoerer, H., Karsch, S., Geissler, M., Veisz, L., Schmid, K., Gallacher, J. G., Jamison, S. P., Jaroszynski, D., Pretzler, G. \& Sauerbrey, R. (2006). Generation of quasimonoenergetic electron bunches with 80 -fs laser pulses, Phys. Rev. Lett. 96(10): 105004.

Hosokai, T., Kinoshita, K., Zhidkov, A., Nakamura, K., Watanabe, T., Ueda, T., Kotaki, H., Kando, M., Nakajima, K. \& Ueseka, M. (2003). Effect of a laser prepulse on a narrow-cone ejection of mev electrons from a gas jet irradiated by an ultrashort laser pulse, Phys. Rev. E 67: 036407.

Khachatryan, A. G., van Goor, F. A., Boller, K.-J., Reitsma, A. J. W. \& Jaroszynski, D. A. (2004). Extremely short relativistic-electron-bunch generation in the laser wakefield via novel bunch injection scheme, Phys. Rev. ST Accel. Beams 7(12): 121301.

Kruer, W. L. (1988). The physics of laser plasma interactions, Addison-Wesley, New-York.

Leemans, W. P., Nagler, B., Gonsalves, A. J., Tòth, C., Nakamura, K., Geddes, C. G. R., Esarey, E., Schroeder, C. B. \& Hooker, S. M. (2006). GeV electron beams from a centimetre-scale accelerator, Nature Physics 2: 696-699.

Leemans, W. P., van Tilborg, J., Faure, J., Geddes, C. G. R., Tóth, C., Schroeder, C. B., Esarey, E., Fubiani, G. \& Dugan, G. (2004). Terahertz emission from laser accelerated electron bunches, Phys. Plasmas 11(5): 2899.

Malka, V., Faure, J., Gauduel, Y. A., Lefebvre, E., Rousse, A. \& Ta Phuoc, K. (2008). Principles 
and applications of compact laserplasma accelerators, Nature Physics 44: 447-453.

Malka, V., Faure, J., Marqus, J.-R., Amiranoff, F., Rousseau, J.-P., Ranc, S., Chambaret, J.-P., Najmudin, Z., Walton, B., Mora, P. \& Solodov, A. (2001). Characterization of electron beams produced by ultrashort (30 fs) laser pulses, Phys. Plasmas 8: 0 .

Malka, V., Fritzler, S., Lefebvre, E., Aléonard, M.-M., Burgy, F., Chambaret, J.-P., Chemin, J.-F., Krushelnick, K., Malka, G., Mangles, S. P. D., Najmudin, Z., Pittman, M., Rousseau, J.-P., Scheurer, J.-N., Walton, B. \& Dangor, A. E. (2002). Electron acceleration by a wake field forced by an intense ultrashort laser pulse, Science 298: 1596-1600.

Malka, V., Lifschitz, A., Faure, J. \& Glinec, Y. (2006). Staged concept of laser-plasma acceleration toward multi-gev electron beams, Phys. Rev. ST Accel. Beams 9(9): 091301.

Mangles, S., Murphy, C. D., Najmudin, Z., Thomas, A. G. R., Collier, J. L., Dangor, A. E., Divall, A. J., Foster, P. S., Gallacher, J. G., Hooker, C. J., Jaroszynski, D. A., Langley, A. J., Mori, W. B., Nooreys, P. A., Viskup, R., Walton, B. R. \& Krushelnick, K. (2004). Mono-energetic beams of relativistic electrons from intense laser plasma interactions, Nature 431: 535.

McKinstrie, C. J. \& Bingham, R. (1992). Stimulated Raman forward scattering and the relativistic modulational instability of light waves in rarefied plasma, Phys. Fluids B 4(8): 2626.

Miura, E., Koyama, K., Kato, S., Saito, S., Adachi, M., Kawada, Y., Nakamura, T. \& Tanimoto, M. (2005). Demonstration of quasi-monoenergetic electron-beam generation in laser-driven plasma acceleration, Appl. Phys. Lett. 86: 251501.

Modena, A., Dangor, A., Najmudin, Z., Clayton, C., Marsh, K., Joshi, C., Malka, V., Darrow, C., Neely, D. \& Walsh, F. (1995). Electron acceleration from the breaking of electron plasma waves, Nature 377: 606-608.

Moore, C., Ting, A., Krushelnick, K., Esarey, E., Hubbard, R., Hafizi, B., Burris, H., Manka, C. \& Sprangle, P. (2004). Electron trapping in self-modulated laser wakefields by raman backscatter, Phys. Rev. Lett. 79: 3909.

Mora, P. (1992). Particle acceleration in a relativistic wave in the adiabatic regime, Phys. Fluids B 4(6): 1630-1634.

Mori, W. B., Joshi, C., Dawson, J. M., Forslund, D. W. \& Kindel, J. M. (1988). Evolution of self-focusing of intense electromagnetic waves in plasma, Phys. Rev. Lett. 60(13): 1298-1301.

Pukhov, A. \& Meyer-ter-Vehn, J. (2002). Laser wake field acceleration: the highly non-linear broken-wave regime, Appl. Phys. B 74: 355-361.

Rechatin, C., Davoine, X., Lifschitz, A., Ismail, A. B., Lim, J., Lefebvre, E., Faure, J. \& Malka, V. (2009). Observation of beam loading in a laser-plasma accelerator, Phys. Rev. Lett. 103(19): 194804.

Rechatin, C., Faure, J., Davoine, X., Lundh, O., Lim, J., Ben-Ismal, A., Burgy, F., Tafzi, A., Lifschitz, A., Lefebvre, E. \& Malka, V. (2010). Characterization of the beam loading effects in a laser plasma accelerator, New Journal of Physics 12(4): 045023.

Rechatin, C., Faure, J., Lifschitz, A., Malka, V. \& Lefebvre, E. (2007). Plasma wake inhibition at the collision of two laser pulses in an underdense plasma, Phys. Plasmas 14(6): 060702.

Sprangle, P. \& Esarey, E. (1992). Interaction of ultrahigh laser fields with beams and plasmas, Phys. Fluids B 4(7): 2241.

Sprangle, P., Tang, C.-H. \& Esarey, E. (1987). Relativistic self-focusing of short-pulse radiation beams in plasmas, IEEE Trans. Plasma Sci. PS-15(2): 145-153.

Strickland, D. \& Mourou, G. (1985). Compression of amplified chirped optical pulses, Opt. 
Comm. 56: 219-221.

Tajima, T. \& Dawson, J. M. (1979). Laser electron accelerator, Phys. Rev. Lett. 43(4): 267.

Ting, A., Esarey, E. \& Sprangle, P. (1990). Nonlinear wake-field generation and relativistic focusing of intense laser pulses in plasmas, Phys. Fluids B 2(6): 1390.

Tsung, F. S., Narang, R., Mori, W. B., Joshi, C., Fonseca, R. A. \& Silva, L. (2004). Near-gev-energy laser-wakefield acceleration of self-injected electrons in a centimeter-scale plasma channel, Phys. Rev. Lett. 93(18): 185002.

Umstadter, D., Chen, S.-Y., Maksimchuk, A., Mourou, G. \& Wagner, R. (1996). Nonlinear optics in plasmas and laser wake field acceleration of electrons, Science 273: 472. 


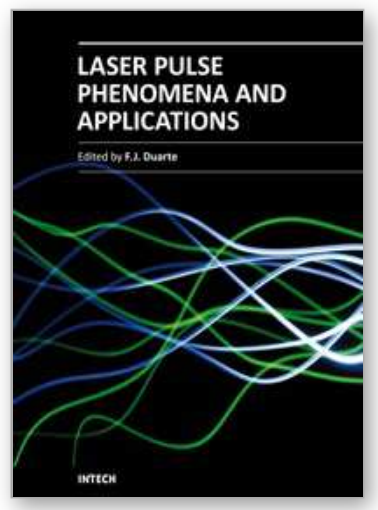

\section{Laser Pulse Phenomena and Applications}

Edited by Dr. F. J. Duarte

ISBN 978-953-307-405-4

Hard cover, 474 pages

Publisher InTech

Published online 30, November, 2010

Published in print edition November, 2010

Pulsed lasers are available in the gas, liquid, and the solid state. These lasers are also enormously versatile in their output characteristics yielding emission from very large energy pulses to very high peak-power pulses.

Pulsed lasers are equally versatile in their spectral characteristics. This volume includes an impressive array of current research on pulsed laser phenomena and applications. Laser Pulse Phenomena and Applications covers a wide range of topics from laser powered orbital launchers, and laser rocket engines, to laser-matter interactions, detector and sensor laser technology, laser ablation, and biological applications.

\section{How to reference}

In order to correctly reference this scholarly work, feel free to copy and paste the following:

Victor Malka (2010). Laser Plasma Accelerators: towards High Quality Electron Beam, Laser Pulse Phenomena and Applications, Dr. F. J. Duarte (Ed.), ISBN: 978-953-307-405-4, InTech, Available from: http://www.intechopen.com/books/laser-pulse-phenomena-and-applications/laser-plasma-acceleratorstowards-high-quality-electron-beam

\section{INTECH}

open science | open minds

\section{InTech Europe}

University Campus STeP Ri

Slavka Krautzeka 83/A

51000 Rijeka, Croatia

Phone: +385 (51) 770447

Fax: +385 (51) 686166

www.intechopen.com

\section{InTech China}

Unit 405, Office Block, Hotel Equatorial Shanghai

No.65, Yan An Road (West), Shanghai, 200040, China

中国上海市延安西路65号上海国际贵都大饭店办公楼 405 单元

Phone: +86-21-62489820

Fax: +86-21-62489821 
(C) 2010 The Author(s). Licensee IntechOpen. This chapter is distributed under the terms of the Creative Commons Attribution-NonCommercialShareAlike-3.0 License, which permits use, distribution and reproduction for non-commercial purposes, provided the original is properly cited and derivative works building on this content are distributed under the same license. 